\title{
Ricci Solitons in $f$-Kenmotsu Manifolds with the semi-symmetric non-metric connection
}

\author{
Tolga Demirli, Cumali Ekici, Ali Gorgulu \\ Eskisehir Osmangazi University, Department of Mathematics-Computer, 26480 Eskisehir-Turkey \\ Received: 25 November 2016, Accepted: 17 December 2016 \\ Published online: 22 December 2016.
}

\begin{abstract}
In this study, some curvature conditions are given for 3-dimensional $f$-Kenmotsu manifolds with the semi-symmetric nonmetric connection. It is showed that this manifold is not always $\xi$-projective flat. Moreover, it is informed that if 3-dimensional $f$ Kenmotsu manifold with the semi-symmetric non-metric connection is Ricci semi-symmetric and regular, then the manifold is an Einstein manifold. Finally, it is proved that 3 -dimensional $f$-Kenmotsu manifold with the semi-symmetric non-metric connection is also an $\eta$-Einstein manifold and the Ricci soliton defined on this manifold is named expanding or shrinking with respect to values of $f$ and $\lambda$ constant.
\end{abstract}

Keywords: Kenmotsu manifolds, Ricci solitons, semi-symmetric non-metric connection.

\section{Introduction}

In 1983, Sharma and Sinha started to study of the Ricci solitons in contact geometry [15]. Later Mukut Mani Tripathi, Cornelia Livia Bejan and Mircea Crasmareanu and others extensively studied Ricci solitons in contact metric manifolds [3], [17]. Kenmotsu studied a class of contact Riemannian manifolds satisfying some special conditions and this manifold is known as Kenmotsu manifolds, in 1972 [7].

The manifold $M$ with $(\phi, \xi, \eta, g)$ structure is said to be normal if $[\phi, \phi]+2 d \eta \otimes \xi=0$ and also it is said to be almost cosymplectic if $d \eta=0$ and $d \Phi=0$. If it is normal and almost cosymplectic, it is called cosymplectic [18]. Olszak and Rosca looked into $f$ - Kenmotsu manifolds, explained in section 2, in a geometric aspect and gave some curvature conditions [11]. Besides this the other mathematicians proved that a Ricci symmetric $f$-Kenmotsu manifold is an Einstein manifold. In 2010, authors show that Ricci semi-symmetric $\alpha$-Kenmotsu manifolds are also Einstein manifolds [12]. As we are talking about $f$-Kenmotsu manifold, we also mean an almost contact metric manifold which is normal and locally conformal almost cosymplectic.

In 2012, Nagaraja ve Premalatha studied exclusively about Ricci solitons on Kenmotsu manifolds [10]. Agashe and Chafle, Liang, Pravonovic and Sengupta, Yıldız and Çetinkaya studied semi-symmetric non-metric connection in different ways [1], [8], [13], [14] and [18].

The paper is organized as follows: After introduction in section 2, we give some basic notions used in this study. In section 3, we introduce $f$-Kenmotsu manifolds. In the next section, we study $f$-Kenmotsu manifolds with the semi-symmetric non-metric connection and show that this manifold is not always $\xi$-projective flat. In section 5, we inform that if 3-dimensional $f$-Kenmotsu manifold with the semi-symmetric non-metric connection is Ricci semi-symmetric and regular, then manifold is also called Einstein manifold. In section 6 , it is proved that $f$-Kenmotsu 
manifold with the semi-symmetric non-metric connection is $\eta$-Einstein manifold and Ricci soliton defined on this manifold is classified with respect to values of $f$ and $\lambda$ constant.

\section{Preliminaries}

Let $M$ be a 3-dimensional differentiable manifold with an almost contact structure $(\phi, \xi, \eta, g)$ satisfying

$$
\begin{array}{ll}
\eta(\xi)=1, \phi \xi=0, & \eta(\phi X)=0, \\
g(X, \phi Y)=-g(\phi X, Y), & \phi^{2}(X)=-X+\eta(X) \xi, \\
g(X, \xi)=\eta(X), & g(\phi X, \phi Y)=g(X, Y)-\eta(X) \eta(Y)
\end{array}
$$

for any vector fields $X, Y \in \chi(M)$, where $\phi$ is a $(1,1)$ tensor field, $\xi$ is a vector field, $\eta$ is a 1 -form and $g$ is the Riemannian metric. Then $M$ is called an almost contact manifold. For an almost contact manifold $M$, it follows that [18]

$$
\begin{aligned}
& \left(\nabla_{X} \phi\right) Y=\nabla_{X} \phi Y-\phi\left(\nabla_{X} Y\right) \\
& \left(\nabla_{X} \eta\right) Y=\nabla_{X} \eta(Y)-\eta\left(\nabla_{X} Y\right)
\end{aligned}
$$

Let $R$ be Riemann curvature tensor, $S$ Ricci curvature tensor, $Q$ Ricci operator and $\left\{e_{1}, \ldots, e_{n}\right\}$ be orthonormal basis of $T_{P}(M) . \forall X, Y \in \chi(M)$ it follows that [6]

$$
\begin{gathered}
S(X, Y)=\sum_{i=1}^{n} g\left(R\left(e_{i}, X\right) Y, e_{i}\right) \\
Q(X)=-\sum_{i=1}^{n} R\left(e_{i}, X\right) e_{i}
\end{gathered}
$$

and

$$
S(X, Y)=g(Q(X), Y)
$$

If the Ricci tensor $S$ of an $f$-Kenmotsu manifold $M$ satisfies the condition

$$
S(X, Y)=\alpha g(X, Y)+\beta \eta(X) \eta(Y)
$$

where $\alpha, \beta$ are certain scalars, then $M$ is said to be $\eta$ Einstein manifold. If $\beta=0$, then $M$ manifold is Einstein manifold $[5]$.

In a three dimensional Riemann manifold the curvature tensor $R$ is described as

$$
R(X, Y) Z=S(Y, Z) X-g(X, Z) Q Y+g(Y, Z) Q X-S(X, Z) Y-\frac{\tau}{2}[g(Y, Z) X-g(X, Z) Y]
$$

where $S$ is the Ricci tensor, $Q$ is the Ricci operator and $\tau$ is the scalar curvature for 3-dimensional $M$ manifold [18].

On the other hand, let $\mathrm{M}$ be an $n$-dimensional Riemannian manifold with the Riemannian connection $\nabla$. A linear connection $\widetilde{\nabla}$ on $M$ is said to be a semi-symmetric connection if its torsion tensor $\widetilde{T}$ of the connection $\widetilde{\nabla}$ satisfies

$$
\widetilde{T}(X, Y)=\eta(Y) X-\eta(X) Y
$$


where $\eta$ is a non-zero 1 -form and $\widetilde{T} \neq 0$.

If moreover $\widetilde{\nabla} g=0$ then the connection is called a semi-symmetric metric connection. If $\widetilde{\nabla} g \neq 0$ then the connection is called a semi-symmetric non-metric [19].

For $n \geq 1, M$ is locally projectively flat if and only if the well known projective curvature tensor $P$ vanishes. Projective curvature tensor $P$ is defined by

$$
P(X, Y) Z=R(X, Y) Z-\frac{1}{2 n}\{S(Y, Z) X-S(X, Z) Y\}
$$

for any $X, Y, Z \in \chi(M)$, where $R$ is the curvature tensor and $S$ is the Ricci tensor of M [9]. If $P(X, Y) \xi=0$ for any $X, Y \in \chi(M), M$ manifold is called $\xi$-projective flat [18].

According to [2], a Ricci soliton is a natural generalization of an Einstein metric and is defined on a Riemannian manifold $(M, g)$. A Ricci soliton is a triple $(g, V, \lambda)$ with $g$ a Riemannian metric, $V$ a vector field, and $\lambda$ a real scalar such that

$$
L_{V} g+2 S+2 \lambda g=0
$$

where $S$ is a Ricci tensor of $M$ and $L_{V}$ denotes the Lie derivative operator along the vector field $V$. The Ricci soliton is said to be shrinking, steady, and expanding accordingly as $\lambda$ is negative, zero, and positive, respectively.

\section{$3 f$-Kenmotsu manifolds}

Let $M$ be a 3-dimensional almost contact manifold. $(M, \phi, \xi, \eta, g)$ is an $f$-Kenmotsu manifold if the covariant differentiation of $\phi$ satisfies [18],

$$
\left(\nabla_{X} \phi\right) Y=f(g(\phi X, Y) \xi-\eta(Y) \phi X)
$$

where $f \in C^{\infty}(M, \mathbb{R})$ such that $d f \wedge \eta=0$. If $f=\alpha=$ constant $\neq 0$, the manifold is said to be an $\alpha$-Kenmotsu. If $f=1$, then 1-Kenmotsu manifold is also called Kenmotsu manifold. If $f^{2}+f^{\prime} \neq 0$, then $f$-Kenmotsu manifold is said to be regular, where $f^{\prime}=\xi f[4]$. By using (1) and (2), it can be shown that

$$
\left(\nabla_{X} \eta\right) Y=f g(\phi X, \phi Y)
$$

From (11), we have [16]

$$
\nabla_{X} \xi=f(X-\eta(X) \xi)
$$

Also from (6), in a 3-dimensional $f$-Kenmotsu manifold we have

$$
R(X, Y) Z=\left(\frac{\tau}{2}+2 f^{2}+2 f^{\prime}\right)(X \wedge Y)-\left(\frac{\tau}{2}+3 f^{2}+3 f^{\prime}\right)\{\eta(X)(\xi \wedge Y) Z+\eta(Y)(X \wedge \xi) Z\}
$$

and

$$
S(X, Y)=\left(\frac{\tau}{2}+f^{2}+f^{\prime}\right) g(X, Y)-\left(\frac{\tau}{2}+3 f^{2}+3 f^{\prime}\right) \eta(X) \eta(Y)
$$

Thus from (15), we get

$$
S(X, \xi)=-2\left(f^{2}+f^{\prime}\right) \eta(X)
$$


where $\tau$ is the scalar curvature of $M$ and $f^{\prime}=\xi f[11]$.

Using (14) and (15), we obtain

$$
\begin{gathered}
R(X, Y) \xi=-\left(f^{2}+f^{\prime}\right)[\eta(Y) X-\eta(X) Y], \\
R(\xi, X) \xi=-\left(f^{2}+f^{\prime}\right)(\eta(X) \xi-X), \\
Q X=\left(\frac{\tau}{2}+f^{2}+f^{\prime}\right) X-\left(\frac{\tau}{2}+3 f^{2}+3 f^{\prime}\right) \eta(X) \xi .
\end{gathered}
$$

It can be easily checked out from (9) by using (17) and (16) that a 3-dimensional $f$-Kenmotsu manifold is always $\xi$ projectively flat [18].

\section{$4 f$-Kenmotsu Manifolds with the semi-symmetric non-metric connection}

Let $\widetilde{\nabla}$ be a linear connection and $\nabla$ be a Riemann connection of an $f$-Kenmotsu manifold $M$. This $\widetilde{\nabla}$ lineer connection defined by

$$
\widetilde{\nabla}_{X} Y=\nabla_{X} Y+\eta(Y) X
$$

where $\eta-1$-form and any vector fields $X, Y \in \chi(M)$, denotes the semi-symmetric non-metric connection [1].

For $f$-Kenmotsu manifold with the semi-symmetric non-metric connection, using (2), (11) and (20) we have

$$
\left(\widetilde{\nabla}_{X} \phi\right) Y=f(g(\phi X, Y) \xi-2 \eta(Y) \phi X)
$$

for any vector fields $X, Y \in \chi(M)$, where $\phi$ is a $(1,1)$ tensor field, $\xi$ is a vector field, $\eta$ is a 1-form and $f \in C^{\infty}(M, \mathbb{R})$ such that $d f \wedge \eta=0$. As a consequence of $d f \wedge \eta=0$, we get

$$
d f=f^{\prime} \text { and } X(f)=f^{\prime} \eta(X)
$$

where $f^{\prime}=\xi f$ [11]. If $f=\alpha=$ constant $\neq 0$, then the manifold is a $\alpha$-Kenmotsu. If $f=0$, then the manifold is cosymplectic. An $f$-Kenmotsu manifold with the semi-symmetric non-metric connection is said to be regular if $f^{2}+f+2 f^{\prime} \neq 0$.

By using (2) and (21), we get

$$
\widetilde{\nabla}_{X} \xi=f(2 X-\eta(X) \xi)
$$

From (2), (20) and (12), we have

$$
\left(\widetilde{\nabla}_{X} \eta\right) Y=f g(\phi X, \phi Y) .
$$

The curvature tensor $\widetilde{R}$ of an $f$-Kenmotsu manifold $M$ with respect to the semi-symmetric non-metric connection $\widetilde{\nabla}$ is defined by

$$
\widetilde{R}(X, Y) \xi=\widetilde{\nabla}_{X} \widetilde{\nabla}_{Y} \xi-\widetilde{\nabla}_{Y} \widetilde{\nabla}_{X} \xi-\widetilde{\nabla}_{[X, Y]} \xi .
$$

With the help of (20), (23) and (13), we get

$$
\widetilde{\nabla}_{X} \widetilde{\nabla}_{Y} \xi=X(f) 2 Y-X(f) \eta(Y) \xi+2 f \nabla_{X} Y-f X \eta(Y) \xi-\eta(Y) f^{2} X+\eta(Y) \eta(X) f^{2} \xi+f \eta(Y) X
$$


and

$$
-\widetilde{\nabla}_{[X, Y]} \xi=-2 f \nabla_{X} Y-2 f \eta(Y) X+2 f \nabla_{Y} X+2 f \eta(X) Y+f X \eta(Y) \xi-f Y \eta(X) \xi .
$$

Using (26) and (27) in (25), we get

$$
\widetilde{R}(X, Y) \xi=X(f) 2 Y-X(f) \eta(Y) \xi-Y(f) 2 X+Y(f) \eta(X) \xi+f^{2} \eta(X) Y-f^{2} \eta(Y) X+f \eta(X) Y-f \eta(Y) X
$$

By using (22) in (28), it follows that

$$
\widetilde{R}(X, Y) \xi=-\left(f^{2}+f+2 f^{\prime}\right)(\eta(Y) X-\eta(X) Y) .
$$

From (29), we have

$$
\widetilde{R}(\xi, Y) \xi=-\left(f^{2}+f+2 f^{\prime}\right)(\eta(Y) \xi-Y)
$$

and

$$
\widetilde{R}(X, \xi) \xi=-\left(f^{2}+f+2 f^{\prime}\right)(X-\eta(X) \xi) .
$$

Taking the inner product with $Z$ in (29), we have

$$
g(\widetilde{R}(X, Y) \xi, Z)=-\left(f^{2}+f+2 f^{\prime}\right)(\eta(Y) g(X, Z)-\eta(X) g(Y, Z))
$$

which is used in the proof of following lemma.

Lemma 1. Let $M$ be a 3-dimensional $f$-Kenmotsu manifold with the semi-symmetric non-metric connection, $\widetilde{S}$ Ricci curvature tensor and $\widetilde{Q}$ Ricci operator. Then

$$
\widetilde{S}(X, \xi)=-2\left(f^{2}+f+2 f^{\prime}\right) \eta(X),
$$

and

$$
\widetilde{Q} \xi=-2\left(f^{2}+f+2 f^{\prime}\right) \xi
$$

Proof. Contracting with $Y$ and $Z$ in (32) and summing over $i=1,2, \ldots, n$, from (3) expression the proof of (33) is completed. Then also using (5) and (1) in (33), the proof of (34) is completed.

Lemma 2. Let $M$ be a 3-dimensional $f$-Kenmotsu manifold with the semi-symmetric non-metric connection, $\tau$ scalar curvature tensor, $\widetilde{S}(X, Y)$ Ricci curvature tensor and $\widetilde{Q} X$ Ricci operator. Then it follows that

$$
\widetilde{S}(X, Y)=\left(\frac{\tau}{2}+f^{2}+f+2 f^{\prime}\right) g(X, Y)-\left(\frac{\tau}{2}+3 f^{2}+3 f+6 f^{\prime}\right) \eta(X) \eta(Y)
$$

and

$$
\widetilde{Q} X=\left(\frac{\tau}{2}+f^{2}+f^{\prime}\right) X-\left(\frac{\tau}{2}+3 f^{2}+3 f^{\prime}\right) \eta(Y) \xi .
$$

Proof. Contracting (31) with $Y$, we get

$$
g(\widetilde{R}(X, \xi) \xi, Y)=-\left(f^{2}+f+2 f^{\prime}\right)(g(X, Y)-\eta(X) \eta(Y))
$$

Using (33), putting $X=\xi, Y=X, Z=Y$ in (7) and contracting with $\xi$, we obtain

$$
\begin{aligned}
\widetilde{R}(\xi, X, Y, \xi)= & \widetilde{S}(X, Y)-2\left(f^{2}+f+2 f^{\prime}\right) g(Y, X)-\frac{\tau}{2}(g(X, Y)-\eta(X) \eta(Y)) \\
& +2\left(f^{2}+f+2 f^{\prime}\right) \eta(X) \eta(Y)+2\left(f^{2}+f+2 f^{\prime}\right) \eta(X) \eta(Y) .
\end{aligned}
$$


With the help of (37) and (38) proof of (35) is completed.

Using (35) and (5), it's verified that

$$
g\left(\widetilde{Q} X-\left[\left(\frac{\tau}{2}+f^{2}+f+2 f^{\prime}\right) X-\left(\frac{\tau}{2}+3 f^{2}+3 f+6 f^{\prime}\right) \eta(X) \xi\right], Y\right)=0 .
$$

Since $Y \neq 0$ in (39), the proof of (36) is completed.

Example 1. (a 3-dimensional $f$-Kenmotsu manifold with the semi-symmetric non-metric connection.) We consider the 3-dimensional manifold $M=\left\{(x, y, z) \in \mathbb{R}^{3}, z \neq 0\right\}$, where $(x, y, z)$ are the standart coordinates in $\mathbb{R}^{3}$. The vector fields

$$
e_{1}=z^{2} \frac{\partial}{\partial x}, e_{2}=z^{2} \frac{\partial}{\partial y}, e_{3}=\frac{\partial}{\partial z}
$$

are linearly independent at each point of $M$. Let $g$ be the Riemannian metric defined by

$$
\begin{aligned}
& g\left(e_{1}, e_{3}\right)=g\left(e_{2}, e_{3}\right)=g\left(e_{1}, e_{2}\right)=0 \\
& g\left(e_{1}, e_{1}\right)=g\left(e_{2}, e_{2}\right)=g\left(e_{3}, e_{3}\right)=1
\end{aligned}
$$

Let $\eta$ be the 1 -form defined by $\eta(Z)=g\left(Z, e_{3}\right)$ for any $Z \in \chi(M)$. Let $\phi$ be the $(1,1)$ tensor field defined by $\phi\left(e_{1}\right)=-e_{2}, \phi\left(e_{2}\right)=e_{1}, \phi\left(e_{3}\right)=0$.

Then using linearity of $\phi$ and $g$ we have

$$
\begin{gathered}
\eta\left(e_{3}\right)=1, \quad \phi^{2}(Z)=-Z+\eta(Z) e_{3} \\
g(\phi Z, \phi W)=g(Z, W)-\eta(Z) \eta(W)
\end{gathered}
$$

for any $Z, W \in \chi(M)$. Now, by direct computations we obtain

$$
\left[e_{1}, e_{2}\right]=0,\left[e_{2}, e_{3}\right]=-\frac{2}{z} e_{2},\left[e_{1}, e_{3}\right]=-\frac{2}{z} e_{1},
$$

By using these above equations we get [18]

$$
\begin{aligned}
& \nabla_{e_{i}} e_{i}=\frac{2}{z} e_{3} \text { and } \nabla_{e_{i}} e_{3}=-\frac{2}{z} e_{i}, i=1,2 \\
& \nabla_{e_{2}} e_{1}=\nabla_{e_{1}} e_{2}=\nabla_{e_{3}} e_{1}=\nabla_{e_{3}} e_{2}=\nabla_{e_{3}} e_{3}=0
\end{aligned}
$$

Now we consider at this example for semi-symmetric non-metric connection. From (20) and (40),

$$
\begin{aligned}
& \widetilde{\nabla}_{e_{i}} e_{i}=\frac{2}{z} e_{3} \text { and } \widetilde{\nabla}_{e_{i}} e_{3}=-\frac{2}{z} e_{i}+e_{i} \\
& \widetilde{\nabla}_{e_{i}} e_{j}=\widetilde{\nabla}_{e_{3}} e_{i}=0 \text { and } \widetilde{\nabla}_{e_{3}} e_{3}=e_{3} .
\end{aligned}
$$

where $i \neq j=1,2$. It's known that

$$
\widetilde{R}(X, Y) Z=\widetilde{\nabla}_{X} \widetilde{\nabla}_{Y} Z-\widetilde{\nabla}_{Y} \widetilde{\nabla}_{X} Z-\widetilde{\nabla}_{[X, Y]} Z
$$


By using (41) and (42) we obtain

$$
\begin{aligned}
& \widetilde{R}\left(e_{i}, e_{3}\right) e_{3}=\left(1-\frac{6}{z^{2}}\right) e_{i}, \widetilde{R}\left(e_{i}, e_{j}\right) e_{3}=0, \\
& \widetilde{R}\left(e_{i}, e_{j}\right) e_{j}=\left(\frac{2}{z}-\frac{4}{z^{2}}\right) e_{i}, \widetilde{R}\left(e_{i}, e_{3}\right) e_{j}=0, \\
& \widetilde{R}\left(e_{3}, e_{i}\right) e_{i}=\left(\frac{2}{z}-\frac{6}{z^{2}}\right) e_{3} .
\end{aligned}
$$

where $i \neq j=1,2$.

From (3) and (43), it's verified that

$$
\widetilde{S}\left(e_{i}, e_{i}\right)=-\frac{10}{z^{2}}+\frac{2}{z}+1, i=1,2 . \text { and } \widetilde{S}\left(e_{3}, e_{3}\right)=-\frac{12}{z^{2}}+\frac{4}{z} .
$$

Now from (9), (43) and (44) it's easily found that

$$
\widetilde{P}\left(e_{1}, e_{2}\right) e_{3}=0, \widetilde{P}\left(e_{i}, e_{3}\right) e_{3}=-\frac{2}{z} e_{i}, i=1,2
$$

This leads to the following:

Lemma 3. A 3-dimensional f-Kenmotsu manifold with the semi-symmetric non-metric connection is not always $\xi$-projectively flat.

\section{Ricci-semisymmetric 3-dimensional $f$-Kenmotsu Manifolds with the semi-symmetric non-metric connection}

An $f$ - Kenmotsu manifold with the semi-symmetric non-metric connection is called as Ricci-semisymmetric if

$$
\widetilde{R}(X, Y) . \widetilde{S}=0
$$

holds on $M$.Then

$$
\widetilde{S}(\widetilde{R}(X, Y) U, V)+\widetilde{S}(U, \widetilde{R}(X, Y) V)=0
$$

In (45) replacing $X$ and $U$ by $\xi$, we get

$$
\widetilde{S}(\widetilde{R}(\xi, Y) \xi, V)+\widetilde{S}(\xi, \widetilde{R}(\xi, Y) V)=0
$$

Then with the help of (30) and (31), it's clarified that

$$
\left(f^{2}+f+2 f^{\prime}\right)[\eta(Y) \widetilde{S}(\xi, V)-\widetilde{S}(Y, V)]+\left(f^{2}+f+2 f^{\prime}\right)[g(Y, V) \widetilde{S}(\xi, \xi)-\eta(V) \widetilde{S}(\xi, Y)]=0
$$

By using (33) in (47), we obtain

$$
\widetilde{S}(Y, V)=-2\left(f^{2}+f+2 f^{\prime}\right) g(Y, V)
$$

This leads to the following:

Theorem 1. Let $M$ be a 3-dimensonal regular, non-cosymplectic $f$-Kenmotsu manifold with the semi-symmetric nonmetric connection is Ricci semi-symmetric. Then manifold is an Einstein manifold. 


\section{Ricci Solitons in $f$-Kenmotsu Manifolds with the semi-symmetric non-metric connection}

Let $M$ be a 3-dimensional $f$-Kenmotsu manifold with the semi-symmetric non-metric connection.and $V$ be pointwise collinear with $\xi$ (i.e. $V=b \xi$, where $b$ is a function). Then $\left(L_{V} g+2 S+2 \lambda g\right)(X, Y)=0$, implies

$$
b g\left(\widetilde{\nabla}_{X} \xi, Y\right)+(X b) \eta(Y)+b g\left(X, \widetilde{\nabla}_{Y} \xi\right)+(Y b) \eta(X)+2 \widetilde{S}(X, Y)+2 \lambda g(X, Y)=0
$$

Using (23) in (49), we get

$$
4 b f g(X, Y)-2 f b \eta(X) \eta(Y)+(X b) \eta(Y)+(Y b) \eta(X)+2 \widetilde{S}(X, Y)+2 \lambda g(X, Y)=0
$$

With the substitution of $Y$ with $\xi$ in (50), it follows that

$$
(X b)+(\xi b) \eta(X)+2 b f \eta(X)-4\left(f^{2}+f+2 f^{\prime}\right) \eta(X)+2 \lambda \eta(X)=0 .
$$

Again replacing $X$ by $\xi$ in (51) shows that

$$
\xi \beta=2\left(f^{2}+f+2 f^{\prime}\right)-b f-\lambda
$$

Putting (52) in (51), we obtain

$$
d b=\left[2\left(f^{2}+f+2 f^{\prime}\right)-b f-\lambda\right] \eta
$$

By applying $d$ on (53), we get

$$
\left[2\left(f^{2}+f+2 f^{\prime}\right)-b f-\lambda\right] d \eta=0
$$

Since $d \eta \neq 0$ from (54), we have

$$
\left[2\left(f^{2}+f+2 f^{\prime}\right)-b f-\lambda\right]=0 .
$$

By using (53) and (55), we obtain that $b$ is a constant. Hence from (50) it is verified

$$
\widetilde{S}(X, Y)=-(2 b f+\lambda) g(X, Y)+b f \eta(X) \eta(Y) .
$$

which implies that $M$ is an $\eta$-Einstein manifold. This leads to the following:

Theorem 2. If in a 3-dimensional $f$-Kenmotsu manifold with the semi-symmetric non-metric connection, the metric $g$ is a Ricci soliton and $V$ is a pointwise collinear with $\xi$, then $V$ is a constant multiple of $\xi$ and $g$ is an $\eta$-Einstein manifold of the form (56) and Ricci soliton is expanding or shrinking according as $\lambda=2\left(f^{2}+f+2 f^{\prime}\right)$-bf is positive or negative.

\section{Conclusion}

In this study, we gave some curvature conditions for 3-dimensional $f$-Kenmotsu manifolds with the semi-symmetric nonmetric connection. We also showed that these manifolds are not always $\xi$-projective flat. If 3-dimensional $f$-Kenmotsu manifold with the semi-symmetric non-metric connection is Ricci semi-symmetric and regular, then the manifold is an Einstein manifold. Finally, we proved that 3-dimensional $f$-Kenmotsu manifold with the semi-symmetric non-metric connection is also an $\eta$-Einstein manifold and the Ricci soliton defined expanding or shrinking on this manifold is named with respect to values of $f$ and $\lambda$ constant. 


\section{Acknowledgements}

We would like to thank Prof. Dr. Ahmet Yıldiz for his guidance and the suggestions. This study was supported by the University of Eskişehir Osmangazi, Scientific Research Project Office through grant (ESOGU-BAP 2013-282).

\section{Competing interests}

The authors declare that they have no competing interests.

\section{Authors' contributions}

All authors have contributed to all parts of the article. All authors read and approved the final manuscript.

\section{References}

[1] Agashe, S. and Chafle, R., 1992, A semi-symmetric non-metric connection on a Riemannian manifold, Indian Journal Pure Applications, 23, 6, 399-409.

[2] Bagewadi, C. S. and Ingalahalli, G., 2012, Ricci solitons in $\alpha$-Sasakain manifolds, ISRN Geometry, 13 p.

[3] Bejan, C.L. and Crasmareanu, M., 2011, Ricci solitons in manifolds with quasi-constant curvature, Publicationes Mathematicae, Debrecen, 78, 1, 235-243.

[4] Călin, C. and Crasmareanu, M., 2010, From the Eisenhart problem to Ricci solitons in $f$-Kenmotsu manifolds, Bulletin of the Malaysian Mathematical Sciences Society, 33, 3, 361-368.

[5] Crasmareanu, M., 2012, Parallel tensors and Ricci solitons in $N(k)$-Quasi Einstein manifolds, Indian Journal of Pure and Applied Mathematics, 43, 4, 359-369.

[6] De, U. C. and Shaikh, A.A., 2007, Differential geometry of manifolds, Alpha Science International, 298 p.

[7] Kenmotsu, K., 1972, A class of almost contact Riemannian manifolds, The Tohoku Mathematical Journal, 24, $93-103$.

[8] Liang, K. C., 1972, A nonoscillation theorem for the superlinear case of second order differential equations, SIAM Journal on Applied Mathematics, 23, 4, 456-459.

[9] Mishra R. S., 1984, Structures on differentiable manifold and their applications, Chandrama Prakasana.

[10] Nagaraja, H. G. and Premalatha, C. R., 2012, Ricci solitons in Kenmotsu manifolds, Journal of Mathematical Analysis, 3, 2 , $18-24$.

[11] Olszak, Z., Rosca, R., 1991, Normal locally conformal almost cosymplectic manifolds, Publicationes Mathematicae Debrecen, 39 , 3, 315-323.

[12] Öztürk, H., Aktan, N. and Murathan, C., 2010, On $\alpha$-Kenmotsu manifolds satisfying some certain conditions, Applied Sciences, 12, 115-126.

[13] Prvanovic, M., 1975, On pseudo metric semi-symmetric connections, Publication De L Institut Mathematic Nouvelle Series, 18, 32, 157-164.

[14] Sengupta, J., De, U. C. and Binh, T. Q., 2000, On a type of semi-symmetric non-metric connection on a Riemannian manifold, Indian Journal of Pure and Applied Mathematics, 31, 1659-1670.

[15] Sharma, R. and B. B. Sinha, 1983, On para-A-Einstein manifolds, Publications De L'institut Mathematique, 34, 48, $211-215$.

[16] Shukla, S. S. and Singh, D. D., 2010, On ( $\xi$ )-Trans-Sasakian manifolds, International Journal of Mathematical Analysis, 4, 49, $2401-2414$.

[17] Tripathi, M. M., 2008, Ricci solitons in contact metric manifolds, http://arxiv. org / abs / 0801.4222, 9 p.

[18] Yıldız, A., De, U. C. and Turan, M., 2013, On 3-dimensional $f$-Kenmotsu manifolds and Ricci solitons, Ukrainian Mathematical Journal, 65, 5, 620-628.

[19] Yıldız, A. and Çetinkaya, A., 2013, Kenmotsu manifolds with the semi-symmetric non-metric connection, preprint. 